\title{
What Else Is Decidable about Integer Arrays?^
}

\author{
Peter Habermehl ${ }^{1}$, Radu Iosif ${ }^{2}$, and Tomáš Vojnar ${ }^{3}$ \\ ${ }^{1}$ LSV, ENS Cachan, CNRS, INRIA; 61 av. du Président Wilson, F-94230 Cachan, France \\ and LIAFA, University Paris 7, Case 7014, 75205 Paris Cedex 13 \\ haberm@liafa.jussieu.fr \\ 2 VERIMAG,CNRS, 2 av. de Vignate, F-38610 Gières, France \\ iosif@imag.fr \\ ${ }^{3}$ FIT BUT, Božetěchova 2, CZ-61266, Brno, Czech Republic \\ vojnar@fit.vutbr.cz
}

\begin{abstract}
We introduce a new decidable logic for reasoning about infinite arrays of integers. The logic is in the $\exists^{*} \forall^{*}$ first-order fragment and allows (1) Presburger constraints on existentially quantified variables, (2) difference constraints as well as periodicity constraints on universally quantified indices, and (3) difference constraints on values. In particular, using our logic, one can express constraints on consecutive elements of arrays (e.g., $\forall i .0 \leq i<n \rightarrow a[i+1]=a[i]-1$ ) as well as periodic facts (e.g., $\forall i . i \equiv_{2} 0 \rightarrow a[i]=0$ ). The decision procedure follows the automata-theoretic approach: we translate formulae into a special class of Büchi counter automata such that any model of a formula corresponds to an accepting run of an automaton, and vice versa. The emptiness problem for this class of counter automata is shown to be decidable as a consequence of earlier results on counter automata with a flat control structure and transitions based on difference constraints.
\end{abstract}

\section{Introduction}

Arrays are a fundamental data structure in computer science. They are used in all modern imperative programming languages. To verify software which manipulates arrays, it is essential to have a sufficiently powerful logic, which can express meaningful program properties, arising as verification conditions within, e.g., inductive invariant checking, or verification of pre- and post-conditions. In order to have an automatic decision procedure for the program verification problems, one needs a decidable logic.

In this paper, we develop a logic of arrays indexed by integer numbers, and having integers as values. To be as general as possible, and also to avoid having to deal explicitly with expressions containing out-of-bounds array accesses, we interpret formulae over both-ways infinite arrays. Bounded arrays can then be conveniently expressed in the logic by restricting indices to be within given bounds.

Properties that are typically of interest about arrays in a program are (existentially quantified) boolean combinations of formulae of the form $\forall \mathbf{i} . G \rightarrow V$ where $G$ is a guard expression containing constraints over the universally quantified index variables $\mathbf{i}$

\footnotetext{
* The work was supported by the French Ministry of Research (RNTL project AVERILES), the Czech Grant Agency (projects 102/07/0322, 102/05/H050), the Czech-French Barrande project 2-06-27, and the Czech Ministry of Education by project MSM 0021630528.
} 
(which often range in between some existentially quantified bounds), and $V$ is a value expression containing constraints over array values. Based on examples, we identified two types of array properties which seem to appear quite often in programs: (1) properties relating consecutive elements of an array, e.g., $\forall i . l_{1} \leq i<l_{2} \rightarrow a[i+1]=a[i]-1$, which states the fact that each value of $a$ between two bounds $l_{1}$ and $l_{2}$ is less than its predecessor by one, (2) properties stating periodic facts, e.g., $\forall i$. $i \equiv_{2} 0 \rightarrow a[i]=0$, stating that all even elements of an array $a$ are equal to 0 .

Without specific syntactic restrictions, a logic with such an expressive power can be easily shown to be undecidable as one can encode histories of computations of a 2counter machine [13] as models of a formula over arrays. From this reduction, one can derive two restrictions leading to decidability. The first restriction forbids references to $a[i]$ and $a[i+1]$ in the same formula, which is considered in the work of Bradley, Manna, and Sipma [5]. The second restriction, considered in this paper, allows only array formulae $\forall \mathbf{i} . G \rightarrow V$ in which $V$ does not contain disjunctions. We have chosen the second option, mainly to retain the possibility of relating consecutive arrays elements, i.e., $a[i]$ and $a[i+1]$, which appears to be important for expressing properties of programs.

We introduce a new logic LIA (Logic on Integer Arrays) in the $\exists^{*} \forall^{*}$ first-order fragment. LIA is essentially the set of existentially quantified boolean combinations of (1) array formulae of the form $\forall \mathbf{i} . \varphi(\mathbf{k}, \mathbf{i}) \rightarrow \psi(\mathbf{k}, \mathbf{i}, \mathbf{a})$ where $\mathbf{i}$ is a set of index variables and $\mathbf{a}$ (resp. k) is a set of existentially quantified array (resp. array-bound) variables, $\varphi$ is a formula on index variables with difference as well as periodicity constraints on variables $\mathbf{i}$ wrt. the array-bounds $\mathbf{k}$, and $\psi$ is a difference constraint on array terms, and (2) Presburger arithmetic formulae on array-bound variables. In [8], we give an example program showing the usefulness of this logic to express verification conditions.

We prove decidability of the logic LIA using the classical idea of the connection between logic and automata [18]: from a formula $\varphi$ of the logic, we build an automaton $A_{\varphi}$ such that $\varphi$ is satisfiable if and only if the language of $A_{\varphi}$ is not empty. Decidability of the logic then follows from decidability of the emptiness problem for the class of automata that is deployed. To this end, we define a new class of counter automata, called FBCA (bi-infinite Flat Büchi Counter Automata). These are counter automata running to infinity in both left and right directions, equipped with a Büchi acceptance condition. For an arbitrary formula $\varphi$ of LIA, we give the construction of the FBCA $A_{\varphi}$ whose runs correspond to models of $\varphi$ : the value of the counter $x_{a}$ at a given point $i$ in an execution of $A_{\varphi}$ corresponds to the value of $a[i]$ in a model of $\varphi$. We prove decidability of LIA by showing that the emptiness problem for FBCA is decidable by extending known results [6/4] on flat counter automata with difference bound constraints.

Related work. In the seminal paper [12], the read and write functions from/to arrays and their logical axioms were introduced. A decision procedure for the quantifier-free fragment of the theory of arrays was presented in [10]. Since then, various decidable logics on arrays have been considered-e.g., [17/1191617]. These logics include working with various predicates (reasoning about sortedness, permutations, etc.) and in terms of various arithmetic (usually Presburger) constraints on array indices and/or values of array entries. However, unlike our logic, most of these works consider quantifier free formulae. In these cases, nested array reads (like $a[a[i]]$ ) are allowed, which is not the case in our logic. 
In [5], an interesting logic within the $\exists^{*} \forall^{*}$ fragment is developed. Unlike our decision procedure based on automata theory, the decision procedure of [5] is based on the fact that the universal quantification can be replaced by a finite conjunction. The result is parameterised in the sense of allowing an arbitrary decision procedure to be used for the data stored in arrays. However, compared to our results, [5] does not allow modulo constraints (allowing to speak about periodicity in the array values), general difference constraints on universally quantified indices (only $i-j \leq 0$ is allowed), nor reasoning about array entries at a fixed distance (i.e., reasoning about $a[i]$ and $a[i+k]$ for a constant $k$ and a universally quantified index $i$ ). The authors of [5] give also interesting undecidability results for extensions of their logic. For example, they show that relating adjacent array values $(a[i]$ and $a[i+1])$, or having nested reads, leads to undecidability.

A restricted form of universal quantification within $\exists^{*} \forall^{*}$ formulae is also allowed in [2], where decidability is obtained based on a small model property. Unlike [5] and our work, [2] allows a hierarchy-restricted form of array nesting. However, similar to the restrictions presented above, neither modulo constraints on indices nor reasoning about array entries at a fixed distance are allowed. A similar restriction not allowing to express properties of consecutive elements of arrays then appears also in [3] where a quite general $\exists^{*} \forall^{*}$ logic on multisets of elements with associated data values is considered.

Remark. For space reasons, all proofs are deferred to [8].

\section{Counter Automata}

Given a formula $\varphi$, we denote by $F V(\varphi)$ the set of its free variables. If we denote a formula as $\varphi\left(x_{1}, \ldots, x_{n}\right)$, we assume $F V(\varphi) \subseteq\left\{x_{1}, \ldots, x_{n}\right\}$. For $\varphi\left(x_{1}, \ldots, x_{n}\right)$, we denote by $\varphi\left[t / x_{i}\right], 1 \leq i \leq n$, the formula in which each occurrence of $x_{i}$ is replaced by a term $t$. Given a formula $\varphi$, we denote by $\models \varphi$ the fact that $\varphi$ is logically valid, i.e., it holds in every structure corresponding to its signature. By $\sigma: \mathbb{Z} \rightarrow \mathbb{Z}, \sigma(n)=n+1$, we denote the successor function on integers. In the following, we work with two sets of arithmetic formulae: difference bound matrices and Presburger arithmetic.

A difference bound matrix (DBM) formula is a conjunction of inequalities of the form $x-y \leq c, x \leq c$, or $x \geq c$ where $c \in \mathbb{Z}$ is a constant. If there is no constraint between $x$ and $y$, we may explicitly write $x-y \leq \infty$. In the following, $\mathbb{Z}^{\infty}$ denotes $\mathbb{Z} \cup\{\infty\}$. Let $\mathbf{z}=\left\{z_{1}, \ldots, z_{n}\right\}$ be a designated set of variables, called parameters. A parametric DBM formula is a conjunction of a DBM formula with atomic propositions of the forms $x \leq$ $f(\mathbf{z})$ or $x \geq f(\mathbf{z})$ where $f$ is a linear combination of parameters, i.e., $f=a_{0}+\sum_{i=1}^{n} a_{i} z_{i}$ for some $a_{i} \in \mathbb{Z}, 0 \leq i \leq n$.

A Presburger arithmetic (PA) formula is a disjunction of conjunctions of either linear constraints of the form $\sum_{i=1}^{n} a_{i} x_{i}+b \geq 0$ or modulo constraints $\sum_{i=1}^{n} a_{i} x_{i}+b \equiv c \bmod d$ where $a_{i}, b, c, d \in \mathbb{Z}, c \geq 0$ and $d>0$, are constants. It is well-known that every formula of the arithmetic of integers with addition $\langle\mathbb{Z}, \geq,+, 0,1\rangle$ can be written in this form due to quantifier elimination [15]. Clearly, every DBM formula is also in PA.

A counter automaton (CA) is a tuple $A=\langle\mathbf{x}, Q, \rightarrow\rangle$ where $\mathbf{x}$ is a finite set of counters ranging over $\mathbb{Z}, Q$ a finite set of control states, and $\rightarrow$ a transition relation given by rules $q \stackrel{\varphi\left(\mathbf{x}, \mathbf{x}^{\prime}\right)}{\longrightarrow} q^{\prime}$ where $\varphi$ is an arithmetic formula relating current values of counters $\mathbf{x}$ to 
their future values $\mathbf{x}^{\prime}$. A configuration of a CA $A$ is a pair $(q, v)$ where $q \in Q$ is a control state, and $v: \mathbf{x} \rightarrow \mathbb{Z}$ is a valuation of the counters in $\mathbf{x}$. For a configuration $c=(q, v)$, we designate by $\operatorname{val}(c)=v$ the valuation of the counters in $c$. A configuration $\left(q^{\prime}, v^{\prime}\right)$ is an immediate successor of $(q, v)$ if and only if $A$ has a transition rule $q \stackrel{\varphi\left(\mathbf{x}, \mathbf{x}^{\prime}\right)}{\longrightarrow} q^{\prime}$ such that $=\varphi\left(v(\mathbf{x}), v^{\prime}\left(\mathbf{x}^{\prime}\right)\right)$. A configuration $c$ is a successor of another configuration $c^{\prime}$ iff there exists a sequence of configurations $c=c_{0} c_{1} \ldots c_{n}=c^{\prime}$ such that, for all $0 \leq i<n$, $c_{i+1}$ is an immediate successor of $c_{i}$. Given two control states $q, q^{\prime} \in Q$, a run of $A$ from $q$ to $q^{\prime}$ is a finite sequence of configurations $c_{0} c_{1} \ldots c_{n}$ with $c_{0}=(q, v), c_{n}=\left(q^{\prime}, v^{\prime}\right)$ for some valuations $v, v^{\prime}: \mathbf{x} \rightarrow \mathbb{Z}$, and $c_{i+1}$ is an immediate successor of $c_{i}$ for all $0 \leq i<n$.

Let $S$ be a set. A bi-infinite sequence of $S$ is a function $\beta: \mathbb{Z} \rightarrow S 1$ We denote by ${ }^{\omega} S^{\omega}$ the set of all bi-infinite sequences over $S$. A bi-infinite Büchi counter automaton (BCA) is a tuple $A=\langle\mathbf{x}, Q, L, R, \rightarrow\rangle$ where $\mathbf{x}$ is a finite set of counters, $Q$ is a finite set of control states, $L, R \subseteq Q$ are the left-accepting and right-accepting states, and $\rightarrow$ is a transition relation defined in the same way as for counter automata.

A run of a BCA $A$ is a bi-infinite sequence of configurations $\ldots c_{-2} c_{-1} c_{0} c_{1} c_{2} \ldots$ such that, for all $i \in \mathbb{Z}, c_{i+1}$ is an immediate successor of $c_{i}$. A run $r$ is left-accepting iff there exists a state $q \in L$ and an infinite decreasing sequence of integers $\ldots<i_{2}<i_{1}<0$ such that, for all $j \in \mathbb{N}$, we have $r\left(i_{j}\right)=\left(q, v_{j}\right)$ for some valuations $v_{j}$ of the counters of $A$. Symmetrically, a run is right-accepting iff there exists a state $q \in R$ and an infinite increasing sequence of integers $0<i_{0}<i_{1}<i_{2}<\ldots$ such that, for all $j \in \mathbb{N}$, we have $r\left(i_{j}\right)=\left(q, v_{j}\right)$ for some valuations $v_{j}$ of the counters of $A$. A run is accepting iff it is both left- and right-accepting. The set of all accepting runs of $A$ is denoted as $\mathcal{R}(A)$. If $r \in \mathcal{R}(A)$ is a run of $A$, we define as $\operatorname{val}(r)=\ldots \operatorname{val}(r(-1)) \operatorname{val}(r(0)) \operatorname{val}(r(1)) \ldots$ the bi-infinite sequence of valuations in $r$, and we let $\mathcal{V}(A)=\{\operatorname{val}(r) \mid r \in \mathcal{R}(A)\}$.

Lemma 1. For any $B C A A$, we have $r \in \mathcal{R}(A)$ if and only if $r \circ \sigma \in \mathcal{R}(A)$.

A control path in a CA (or BCA) $A$ is a finite sequence $q_{0} q_{1} \ldots q_{n}$ of control states such that, for all $0 \leq i<n$, there exists a transition rule $q_{i} \stackrel{\varphi_{i}}{\longrightarrow} q_{i+1}$. A cycle is a control path starting and ending in the same control state. An elementary cycle is a cycle in which each state appears only once, except the first one that appears twice. A CA (or BCA) is said to be flat iff each control state belongs to at most one elementary cycle.

Decidability and Closure Properties of FBCA. We consider in the following the class of bi-infinite Büchi counter automata which are flat, whose elementary cycles are labelled with parametric DBM formulae, and the remaining transitions are labelled with PA formulae. Moreover, each transition constraint enforces the values of parameters to remain constant. We call this class FBCA. We prove that the emptiness problem for FBCA is decidable using results of [6]4] and their extensions that can be found in [8].

Lemma 2. The emptiness problem is decidable for the class of FBCA.

\footnotetext{
${ }^{1}$ In the early literature [14], a bi-infinite sequence is defined as the equivalence class of all compositions $\beta \circ \sigma^{n} \circ \sigma^{-m}$ for arbitrary $n, m \in \mathbb{N}$. This is because a bi-infinite sequence remains the same if shifted left or right. For simplicity, we formally distinguish here the bi-infinite sequences $\beta, \beta \circ \sigma^{n}$, and $\beta \circ \sigma^{-n}$ for $n>0$.
} 
The FBCA class is also effectively closed under union and intersection. However, before proceeding, we need to elucidate the meaning of these operations for CA (BCA). For a valuation $v: \mathbf{x} \rightarrow \mathbb{Z}$, if $\mathbf{z} \subseteq \mathbf{x}$ is a subset of the counters in $\mathbf{x}$, let $v \downarrow_{\mathbf{z}}$ denote the restriction of $v$ to the domain $\mathbf{z}$. For some subset $\mathbf{z} \subset \mathbf{x}$ of the counters of $A$ and $s \in \mathcal{V}(A)$, we define the restriction operator on sequences $s \downarrow_{\mathbf{z}}=\ldots \operatorname{val}(s(-1)) \downarrow_{\mathbf{z}} \operatorname{val}(s(0)) \downarrow_{\mathbf{z}}$ $\operatorname{val}(s(1)) \downarrow_{\mathbf{z}} \ldots$, and $\mathcal{V}(A) \downarrow_{\mathbf{z}}=\left\{s \downarrow_{\mathbf{z}} \mid s \in \mathcal{V}(A)\right\}$. Symmetrically, for $\mathbf{z} \supset \mathbf{x}$, we define the extension operator on sequences $\mathcal{V}(A) \uparrow_{\mathbf{z}}=\left\{v \in{ }^{\omega}(\mathbf{z} \mapsto \mathbb{Z})^{\omega} \mid v \downarrow_{\mathbf{x}} \in \mathcal{V}(A)\right\}$.

A class of counter automata is said to be closed under union and intersection if there exist operations $\uplus$ and $\otimes$ such that, for any two FBCA $A_{i}=\left\langle\mathbf{x}_{\mathbf{i}}, Q_{i}, L_{i}, R_{i}, \rightarrow_{i}\right\rangle$, $i=1,2$, we have that $\mathcal{V}\left(A_{1} \uplus A_{2}\right)=\mathcal{V}\left(A_{1}\right) \uparrow_{\mathbf{x}_{\mathbf{1}} \cup \mathbf{x}_{\mathbf{2}}} \cup \mathcal{V}\left(A_{2}\right) \uparrow_{\mathbf{x}_{1} \cup \mathbf{x}_{\mathbf{2}}}$ and $\mathcal{V}\left(A_{1} \otimes A_{2}\right)=$ $\mathcal{V}\left(A_{1}\right) \uparrow_{\mathbf{x}_{1} \cup \mathbf{x}_{2}} \cap \mathcal{V}\left(A_{2}\right) \uparrow_{\mathbf{x}_{1} \cup \mathbf{x}_{2}}$, respectively. The class is said to be effectively closed under union and intersection if these operators are effectively computable.

Proposition 1. Let $A=\langle\mathbf{x}, Q, L, R, \rightarrow\rangle$ be a FBCA. Let $A^{c}=\left\langle\mathbf{x}, Q, L^{c}, R^{c}, \rightarrow\right\rangle$ be the $F B C A$ such that (1) for all $q \in L$ and $q^{\prime} \in Q, q^{\prime}$ belongs to the same elementary cycle as $q$ iff $q^{\prime} \in L^{c}$, (2) for all $q \in R$ and $q^{\prime} \in Q, q^{\prime}$ belongs to the same elementary cycle as $q$ iff $q^{\prime} \in R^{c}$. Then we have that $\mathcal{R}(A)=\mathcal{R}\left(A^{c}\right)$.

Assuming w.l.o.g. that $Q_{1} \cap Q_{2} \neq \emptyset$, the union is defined as $A_{1} \uplus A_{2}=\left\langle\mathbf{x}_{\mathbf{1}} \cup \mathbf{x}_{\mathbf{2}}, Q_{1} \cup\right.$ $\left.Q_{2}, L_{1} \cup L_{2}, R_{1} \cup R_{2}, \rightarrow_{1} \cup \rightarrow_{2}\right\rangle$. The product is defined as $A_{1} \otimes A_{2}=\left\langle\mathbf{x}_{\mathbf{1}} \cup \mathbf{x}_{2}, Q_{1} \times\right.$ $\left.Q_{2}, L_{1}^{c} \times L_{2}^{c}, R_{1}^{c} \times R_{2}^{c}, \rightarrow\right\rangle$ where $\rightarrow$ is as follows: $\left(q_{1}, q_{1}^{\prime}\right) \stackrel{\varphi_{1} \wedge \varphi_{2}}{\longrightarrow}\left(q_{2}, q_{2}^{\prime}\right)$ iff $q_{1} \stackrel{\varphi_{1}}{\longrightarrow} q_{2}$ is a transition rule of $A_{1}$ and $q_{1}^{\prime} \stackrel{\varphi_{2}}{\longrightarrow} q_{2}^{\prime}$ is a transition rule of $A_{2}$. Here, $L_{i}^{c}$ and $R_{i}^{c}$ denote the extended left-accepting and right-accepting sets of $A_{i}$ from Proposition 1 for $i=1,2$.

Lemma 3. The class of FBCA is effectively closed under union and intersection.

\section{A Logic for Integer Arrays}

In this section we define the Logic of Integer Arrays (LIA) that we use to specify properties of programs handling arrays of integers.

Syntax. We consider three types of variables. The array-bound variables $(k, l)$ appear within the so-called array-bound terms. These terms can be used to define intervals of indices and also as static references inside arrays. The index $(i, j)$ and array $(a, b)$ variables are used to build array terms. Fig. 11 shows the syntax of the logic LIA. We use the symbol $T$ to denote the boolean value true. In the following, we will use $f \leq i \leq g$ instead of $f \leq i \wedge i \leq g, i<f$ instead of $i \leq f-1$, and $i=f$ instead of $f \leq i \leq f$. Intuitively, our logic is the set of existentially quantified boolean combinations of:

1. Array formulae of the form $\forall \mathbf{i} . \varphi(\mathbf{k}, \mathbf{i}) \rightarrow \psi(\mathbf{k}, \mathbf{i}, \mathbf{a})$ where $\mathbf{k}$ is a set of array-bound variables, $\mathbf{i}$ is a set of index variables, $\mathbf{a}$ is a set of array variables, $\varphi$ is an arithmetic formula on index variables, and $\psi$ is an arithmetic formula on array terms. In particular, $\psi$ is a DBM formula, and $\varphi$ is composed of atomic propositions of the form either $f \leq i, i \leq f, i-j \leq n$, or $i \equiv_{s} t$ where $f$ is a linear combination of array-bound variables, $n \in \mathbb{Z}$, and $0 \leq t<s$. Both $\mathbf{k}$ and a variables are free in the array formulae, but they can be existentially quantified at the top-most level.

2. PA formulae on array-bound variables. 


$\begin{array}{lll}n, m, s, t \ldots & \in \mathbb{Z} \\ k, l, \ldots & \in & \text { BVar } \\ i, j, \ldots & \in & \text { IVar } \\ a, b, \ldots & \in & \text { AVar } \\ B & :=n|k| B+B \mid B-B \\ I & :=i \mid I+n \\ A & :=a[I] \mid a[B] \\ G & :=B \leq I|I \leq B| I-I \leq n\left|I \equiv_{s} t\right| G \vee G \mid G \wedge G \\ V & :=A \leq B|B \leq A| A-A \leq n \mid V \wedge V \\ C & :=B \leq n \mid B \equiv_{s} t \\ P & :=\top \rightarrow V|G \rightarrow V| \forall i . P \\ U & :=P|C| \neg U|U \vee U| U \wedge U \\ F & :=U|\exists k . F| \exists a . F\end{array}$

constants $(0 \leq t<s)$ array-bound variables index variables array variables array-bound terms index terms array terms guard expressions value expressions array-bound constraints array properties universal formulae LIA formulae

Fig. 1. Syntax of the logic LIA

Examples. To accustom the reader with the logic, we consider several properties of interest that can be stated about arrays. For instance, a strictly increasing ordering of an array $a$ up to a certain bound is defined as $\exists k \forall i .0 \leq i<k \rightarrow a[i]-a[i+1] \leq-1$. The fact that the first $k$ elements of an array $a$ are below the first $l$ elements of an array $b$ at distance 5 is defined as $\exists k, l \forall i, j .0 \leq i<k \wedge 0 \leq j<l \rightarrow a[i]-b[j] \leq-5$. Equality of two arrays up to a certain bound can be expressed as $\exists n \forall i .0 \leq i<n \rightarrow a[i]=b[i]$. The use of modulo constraints as guards for indices allows one to express periodic facts, e.g., $\forall i, j . i \equiv_{2} 0 \wedge j \equiv_{2} 1 \rightarrow a[i] \leq a[j]$ meaning that any value at some even position is less than or equal to any value at some odd position in $a$. In [8], we show that to prove the correctness of an array merging program, such properties are needed.

Semantics. The logic LIA is interpreted on both-ways infinite arrays. This allows us to conveniently deal with out-of-bound reference situations common in programs handling arrays. One can prevent and/or check for out-of-bound references by introducing explicit existentially quantified array-bound variables for array variables. Let $\varphi(\mathbf{k}, \mathbf{a})$ be any LIA formula. A valuation is a pair of partial function $\hat{2}^{2}\langle\mathbf{l}, \mu\rangle$ with $\imath: B \operatorname{Var} \cup I$ Var $\rightarrow \mathbb{Z}_{\perp}$ associating an integer value with every free integer variable and $\mu: A V a r \rightarrow{ }^{\omega} \mathbb{Z}_{\perp}^{\omega}$ associating a bi-infinite sequence of integers with every array symbol $a \in \mathbf{a}$. The valuation $\mathrm{t}$ is extended in the standard way to array-bound terms $(\mathrm{l}(B))$ and index terms $(l(I))$. By $I_{\mathrm{l}, \mu}(A)$, we denote the value of the array term $A$ given by the valuation $\langle\mathbf{l}, \mu\rangle$. The semantics of a formula $\varphi$ is defined in terms of the forcing relation $\models$ as follows:

$$
\begin{array}{rlc}
\langle\mathrm{l}, \mu\rangle \models A \leq B & \Longleftrightarrow & I_{\mathrm{l}, \mu}(A) \leq \mathrm{l}(B) \\
I_{\mathrm{l}, \mu}(a[I])=\mu(a)(\mathrm{l}(I)) \quad\langle\mathrm{l}, \mu\rangle \models A_{1}-A_{2} \leq n & \Longleftrightarrow \quad I_{\mathrm{l}, \mu}\left(A_{1}\right)-I_{\mathrm{l}, \mu}\left(A_{2}\right) \leq n \\
I_{\mathrm{l}, \mu}(a[B])=\mu(a)(\mathrm{l}(B)) \quad\langle\mathrm{l}, \mu\rangle \models \forall i . G \rightarrow V & \Longleftrightarrow \forall n \in \mathbb{Z} \cdot\langle\mathrm{l}[i \leftarrow n], \mu\rangle \models G \rightarrow V \\
\langle\mathrm{l}, \mu\rangle \models \exists a . \psi & \Longleftrightarrow \exists \beta \in{ }^{\omega} \mathbb{Z}^{\omega} \cdot\langle\mathrm{l}, \mu[a \leftarrow \beta]\rangle \models \psi
\end{array}
$$

For space reasons, we do not give here a full definition. However, the missing rules are standard in first-order arithmetic. A model of $\varphi(\mathbf{k}, \mathbf{a})$ is a valuation $\langle\mathbf{l}, \mu\rangle$ such that

\footnotetext{
${ }^{2}$ The symbol $\perp$ is used to denote that a partial function is undefined at a given point.
} 
the formula obtained by interpreting each variable $k \in \mathbf{k}$ as $\mathbf{\imath}(k)$ and each array variable $a \in \mathbf{a}$ as $\mu(a)$ is logically valid: $\langle\mathbf{l}, \mu\rangle \models \varphi$. We define $[\varphi \varphi]=\{\langle\mathbf{l}, \mu\rangle|\langle\mathbf{l}, \mu\rangle|=\varphi\}$. A formula is satisfiable if and only if $[[\varphi]] \neq 0$.

An Undecidability Result. The reason behind the restriction that array terms may not occur within disjunctions in value expressions (cf. Fig. 1) is that, without it, the logic becomes undecidable. The essence of the proof is that an array formula $\forall \mathbf{i} . G \rightarrow$ $V_{1} \vee \ldots \vee V_{n}$, for $n>1$, corresponds to $n$ nested loops in a counter automaton. Undecidability is shown by reduction from the halting problem for 2-counter machines [13].

Lemma 4. The logic obtained by extending LIA with disjunctions within the value expressions is undecidable.

Note that having more than one nested loop is a necessary condition for undecidability of 2-counter machines since a flat 2-counter machine would trivially fall into the class of decidable counter machines from [6]4].

\section{Decidability of the Satisfiability Problem}

The idea behind our method for deciding the satisfiability problem for LIA is that, for any formula of LIA, there exists an FBCA $A_{\varphi}$ such that $\varphi$ has a model if and only if $A_{\varphi}$ has an accepting run. More precisely, each array variable in $\varphi$ has a corresponding counter in $A_{\varphi}$, and given any model of $\varphi$ that associates integer values to all array entries, $A_{\varphi}$ has a run such that the values of the counters at different points of the run match the values of the array entries at corresponding indices in the model. Since, by Lemma 2 the emptiness problem is decidable for FBCA, this leads to decidability of LIA.

In order to build an automaton from a LIA formula, we first normalise it into an existentially quantified positive boolean combination of simple array property formulae (cf. Fig. 11. Second, each such array property formula is translated into an FBCA. The final automaton $A_{\varphi}$ is defined recursively on the structure of the normalised formula with the $\uplus$ and $\otimes$ operators being the counterparts for the $\vee$ and $\wedge$ connectives, respectively.

\subsection{Normalisation of Formulae}

The goal of this step is to transform any formula written using the syntax of Figure 1 into a formula of the following normal form:

$$
\exists \mathbf{k} \exists \mathbf{a} \cdot \bigvee_{c}\left(\bigwedge_{d} \phi_{c d}(\mathbf{a}, \mathbf{k})\right) \wedge \theta_{c}(\mathbf{k})
$$

where $\mathbf{a}$ is a set of array variables, $\mathbf{k}$ is a set of integer variables, and

- $\theta_{d}$ is a conjunction of terms of the forms (i) $g(\mathbf{k}) \geq 0$ or (ii) $g(\mathbf{k}) \equiv_{s} t$ with $g$ being a linear combination of the variables in $\mathbf{k}$ and $0 \leq t<s$,

- $\phi_{c d}$ is a formula of the following forms for $\sim \in\{\leq, \geq\}, m \in \mathbb{N}, 0 \leq t<s, 0 \leq v<u$, $q \in \mathbb{Z}$, and $f_{k}, g_{l}, f_{k}^{1}, g_{l}^{1}, f_{k}^{2}, g_{l}^{2}$ being linear combinations of array-bound variables:

$$
\forall i . \bigwedge_{k=1}^{K} f_{k} \leq i \wedge \bigwedge_{l=1}^{L} i \leq g_{l} \wedge i \equiv_{s} t \rightarrow a[i] \sim h(\mathbf{k})
$$


The (F1) formulae bind all values of $a$ in some interval by some linear combination $h$ of variables in $\mathbf{k}$.

$$
\forall i . \bigwedge_{k=1}^{K} f_{k} \leq i \wedge \bigwedge_{l=1}^{L} i \leq g_{l} \wedge i \equiv_{s} t \rightarrow a[i]-b[i+p] \sim q
$$

Here, $p \in \mathbb{Z}$. The (F2) formulae relate all values of $a$ and $b$ in the same interval such that the distance between the indices of $a$ and $b$, respectively, is constant.

$$
\begin{gathered}
\forall i, j \cdot \bigwedge_{k=1}^{K_{1}} f_{k}^{1} \leq i \wedge \wedge_{l=1}^{L_{1}} i \leq g_{l}^{1} \wedge \wedge_{k=1}^{K_{2}} f_{k}^{2} \leq j \wedge \wedge_{l=1}^{L_{2}} j \leq g_{l}^{2} \wedge \\
i-j \leq p \wedge i \equiv_{s} t \wedge j \equiv_{u} v \rightarrow a[i]-b[j] \sim q
\end{gathered}
$$

Here, $p \in \mathbb{Z}^{\infty}$. The (F3) formulae relate all values of $a$ with all values of $b$ within two (possibly equal) intervals. The case when $p=\infty$ corresponds to the situation when no constraint $i-j \leq p$ with $p \in \mathbb{Z}$ is used.

Lemma 5. A formula of LIA can be equivalently written in the form (NF).

In the following, we refer to the matrix of $\varphi$ as to the formula obtained by forgetting the existential quantifier prefix from the (NF) form of $\varphi$.

\subsection{Formulae and Constraint Graphs}

In [6] ], the set of runs of a flat counter automaton is represented by an unbounded constraint graph. Here, we view the models of a formula as a constraint graph both left- and right-infinite. These constraint graphs are then seen as executions of FBCA, relating in this way models of formulae to runs of automata.

Let $\varphi(\mathbf{k}, \mathbf{a})$ be a formula of type $(\mathrm{F} 1)-(\mathrm{F} 3)$, and $\mathbf{\imath}: \mathbf{k} \rightarrow \mathbb{Z}$ a valuation of its arraybound variables $\mathbf{k}$. For the rest of this section, we fix the valuation $\mathbf{l}$, and we denote by $\varphi_{\mathrm{l}}$ the formula obtained from $\varphi$ by replacing each occurrence of $k \in \mathbf{k}$ by the value $\mathbf{\imath}(k)$.

The formula $\varphi_{\imath}$ can thus be represented by a weighted directed graph $G_{1, \varphi}$ in which each node $(a, n)$ represents the array entry $a[n]$ for some $a \in \mathbf{a}$ and $n \in \mathbb{Z}$, and there is a path of weight $w$ between nodes $(a, n)$ and $(b, m)$ iff the constraint $a[n]-b[m] \leq w$ is implied by $\varphi_{\imath}$. In the next section, we will show that these graphs are in a one-to-one correspondence with the accepting runs of an FBCA.

In order to build the constraint graph of a formula, one needs to pay attention to the following issue. Consider, e.g., the formula $\forall i, j . i-j \leq 3 \wedge i \equiv_{2} 0 \wedge j \equiv_{2} 1 \rightarrow a[i]-$ $b[j] \leq 5$. The constraint graph of this formula needs to have a path of weight 5 between, e.g., $a[0]$ and $b[1], a[0]$ and $b[3], a[0]$ and $b[5]$, etc. As one can easily notice, the span of such paths is potentially unbounded. Since we would like this graph to represent a computation of a flat counter automaton, it is essential to define it as a sequence composed of (a possibly unbounded number of) repetitions of a finite number of (finite) sub-graphs (see, e.g., Fig. 2(a) or Fig. 2(b)]. To this end, we introduce intermediary nodes which are connected between themselves with 0 arcs such that, for each nonlocal constraint of the form $a[n]-b[m] \leq w$ where $|n-m|$ can be arbitrarily large, there exists exactly one path of weight $w$ through these nodes. E.g., in Fig.2(a) there is a path 
$(a, 0) \stackrel{5}{\rightarrow}\left(t_{\varphi},-3\right) \stackrel{0}{\rightarrow} \ldots \stackrel{0}{\rightarrow}\left(t_{\varphi}, 1\right) \stackrel{0}{\rightarrow}(b, 1)$ for the constraint $a[0]-b[1] \leq 5$, another path $(a, 0) \stackrel{5}{\rightarrow}\left(t_{\varphi},-3\right) \stackrel{0}{\rightarrow} \ldots \stackrel{0}{\rightarrow}\left(t_{\varphi}, 3\right) \stackrel{0}{\rightarrow}(b, 3)$ for the constraint $a[0]-b[3] \leq 5$, etc.

Formally, the constraint graph $G_{1, \varphi}=\langle V, E\rangle$ of a formula $\varphi$ of type (F1)-(F3) is defined as follows: The set of vertices is $V=(\mathcal{A} \cup \mathcal{T} \cup\{\zeta\}) \times \mathbb{Z}$. Here, $\mathcal{A}=\{a\}$ for (F1) formulae, and $\mathcal{A}=\{a, b\}$ for (F2)-(F3) formulae, with $a$ or $a, b$ being the arrays that appear in $\varphi$ of type (F1) or (F2)-(F3), respectively. Next, $\mathcal{T}=\emptyset$ for (F1)-(F2) formulae, and $\mathcal{T}=\left\{t_{\varphi}\right\}$ for (F3) formulae where $t_{\varphi}$ is a unique auxiliary symbol (track) associated with each formula $\varphi$ of type (F3). Finally, $\zeta$ is a special shared symbol (zero track). The set of edges $E$ is defined based on the type (F1)-(F3) of $\varphi$. For space reasons, we give here only the definitions for formulae of type (F3), which are the most interesting. Formulae (F1) and (F2) are treated in [8]. In general, for all types of formulae, we have:

$$
E \supset\{(\zeta, k) \stackrel{0}{\rightarrow}(\zeta, k+1) \mid k \in \mathbb{Z}\} \cup\{(\zeta, k+1) \stackrel{0}{\rightarrow}(\zeta, k) \mid k \in \mathbb{Z}\}
$$

i.e., the value of the zero track stays constant.

Constraint graphs for (F3) formulae. Let $\varphi$ be the formula below where $0 \leq s<t$, $0 \leq u<v, p \in \mathbb{Z}^{\infty}, q \in \mathbb{Z}$, and $f_{k}^{1}, g_{l}^{1}, f_{k}^{2}, g_{l}^{2}$ are linear combinations of array-bound variables:

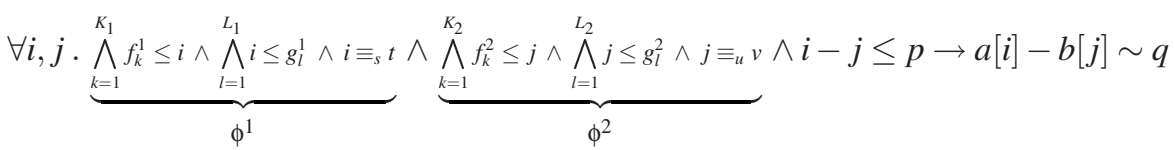

Let $\phi^{1}(i, \mathbf{k})$ and $\phi^{2}(j, \mathbf{k})$ be the subformulae defining the ranges of $i$ and $j$, respectively, and $\mathcal{P}_{1}^{1}=\left\{n \in \mathbb{Z} \mid \models \phi_{1}^{1}[n / i]\right\}$ and $\mathcal{P}_{1}^{2}=\left\{n \in \mathbb{Z} \mid \models \phi_{1}^{2}[n / j]\right\}$ be these ranges under the valuation $\mathrm{l}$. Let $T_{\leq}=\left\{\left(t_{\varphi}, k\right) \stackrel{0}{\rightarrow}\left(t_{\varphi}, k+1\right) \mid k \in \mathbb{Z} \wedge \exists n \in \mathcal{P}_{1}^{1} \exists m \in \mathcal{P}_{1}^{2} . n-m \leq p\right\}$ and $T_{\geq}=\left\{\left(t_{\varphi}, k\right) \stackrel{0}{\rightarrow}\left(t_{\varphi}, k-1\right) \mid k \in \mathbb{Z} \wedge \exists n \in \mathcal{P}_{1}^{1} \exists m \in \mathcal{P}_{1}^{2} . n-m \geq p\right\}$. Note that $T_{\leq}$and $T_{\geq}$are empty if the precondition of $\varphi$ is not satisfiable. The set of edges $E$ is defined by the following case split:

1. If $p<\infty$, we consider two cases based on the direction of $a[i]-b[j] \sim q$ :

(a) for $a[i]-b[j] \leq q$, we have (Fig. 2(a) $]$ :

$$
E \supset\left\{(a, k) \stackrel{q}{\rightarrow}\left(t_{\varphi}, k-p\right) \mid k \in \mathcal{P}_{1}^{1}\right\} \cup\left\{\left(t_{\varphi}, k\right) \stackrel{0}{\rightarrow}(b, k) \mid k \in \mathcal{P}_{1}^{2}\right\} \cup T_{\leq}
$$

(b) for $a[i]-b[j] \geq q$, we have:

$$
E \supset\left\{(b, k) \stackrel{-q}{\longrightarrow}\left(t_{\varphi}, k+p\right) \mid k \in \mathcal{P}_{1}^{2}\right\} \cup\left\{\left(t_{\varphi}, k\right) \stackrel{0}{\rightarrow}(a, k) \mid k \in \mathcal{P}_{1}^{1}\right\} \cup T_{\geq}
$$

2. If $p=\infty$, we consider again two cases based on the direction of $a[i]-b[j] \sim q$ :

(a) for $a[i]-b[j] \leq q$, we have (Fig. 2(b)]:

$$
E \supset\left\{(a, k) \stackrel{q}{\rightarrow}\left(t_{\varphi}, k\right) \mid k \in \mathcal{P}_{1}^{1}\right\} \cup\left\{\left(t_{\varphi}, k\right) \stackrel{0}{\rightarrow}(b, k) \mid k \in \mathcal{P}_{1}^{2}\right\} \cup T_{\leq} \cup T_{\geq}
$$

(b) for $a[i]-b[j] \geq q$, we have:

$$
E \supset\left\{(b, k) \stackrel{-q}{\longrightarrow}\left(t_{\varphi}, k\right) \mid k \in \mathcal{P}_{1}^{2}\right\} \cup\left\{\left(t_{\varphi}, k\right) \stackrel{0}{\rightarrow}(a, k) \mid k \in \mathcal{P}_{1}^{1}\right\} \cup T_{\leq} \cup T_{\geq}
$$

Nothing else is in $E$. 

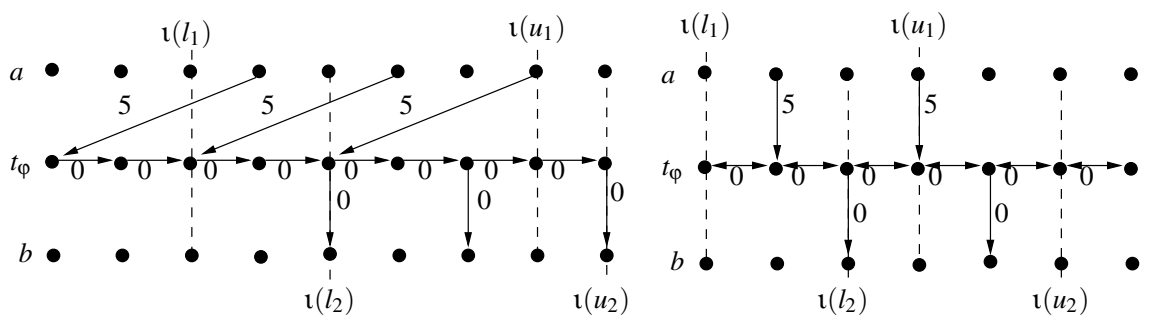

(a) $\forall i, j . l_{1} \leq i \leq u_{1} \wedge l_{2} \leq j \leq u_{2} \wedge i-j \leq 3 \wedge i \equiv_{2}$ $0 \wedge j \equiv_{2} 1 \rightarrow a[i]-b[j] \leq 5$

(b) $\forall i, j . l_{1} \leq i \leq u_{1} \wedge l_{2} \leq j \leq u_{2} \wedge i \equiv_{2}$ $0 \wedge j \equiv_{2} 1 \rightarrow a[i]-b[j] \leq 5$

Fig. 2. Examples of constraint graphs for (F3) formulae

Relating constraint graphs and models of formulae. We can now prove a correspondence between constraint graphs and models of formulae of the forms (F1)-(F3). Namely, it is the fact that if the vertices of a constraint graph for a formula $\varphi$ can be labelled in a consistent way, then from the labelling, one can extract a model for $\varphi$, and vice versa. This formalises correctness of the construction for constraint graphs using the additional tracks.

Let $\varphi(\mathbf{k}, \mathbf{a})$ be a formula of the forms (F1)-(F3), $\mathbf{\imath}: \mathbf{k} \rightarrow \mathbb{Z}$ a valuation of the arraybound variables in $\varphi$, and $G_{1, \varphi}=(V, E)$ its corresponding constraint graph. A labelling $L a b: V \rightarrow \mathbb{Z}$ of $G_{1, \varphi}$ is called consistent if and only if (1) for all edges $v_{1} \stackrel{k}{\rightarrow} v_{2} \in E$, we have $\operatorname{Lab}\left(v_{1}\right)-\operatorname{Lab}\left(v_{2}\right) \leq k$ and (2) $\operatorname{Lab}((\zeta, n))=0$ for all $n \in \mathbb{Z}$.

Lemma 6. Let $\varphi(\mathbf{k}, \mathbf{a})$ be a formula of the form (F1)-(F3). Then, for all valuations $\mathbf{\imath}: \mathbf{k} \rightarrow \mathbb{Z}$ and $\mu: \mathbf{a} \rightarrow{ }^{\omega} \mathbb{Z}^{\omega}$, we have that $\langle\mathbf{\imath}, \mu\rangle \models \varphi$ if and only if there exists a consistent labelling Lab of $G_{\mathrm{l}, \varphi}$ such that $\mu(a)(i)=\operatorname{Lab}((a, i))$ for all $a \in \mathbf{a}$ and $i \in \mathbb{Z}$.

\subsection{From Formulae to Counter Automata}

In this section, we describe the construction of an $\mathrm{FBCA} A_{\varphi}$ corresponding to a formula $\varphi$ such that (1) each run of $A_{\varphi}$ corresponds to a model of $\varphi$, and (2) for each model of $\varphi$, $A_{\varphi}$ has at least one corresponding run. In this way, we effectively reduce the satisfiability problem for LIA to the emptiness problem for FBCA.

The construction of FBCA is by induction on the structure of the formulae. For the rest of this section, let $\varphi$ be a formula, $\mathbf{k}$ the set of array-bound variables in $\varphi$, and $\mathbf{a}$ the set of array variables in $\varphi$, i.e., $F V(\varphi)=\mathbf{k} \cup \mathbf{a}$. Suppose that $\varphi$ is the matrix of a formula in the normal form (NF), i.e., $\varphi: \bigvee_{i \in I} \theta_{i}(\mathbf{k}) \wedge \bigwedge_{j \in J} \psi_{i j}(\mathbf{k}, \mathbf{a})$ where $\theta_{i}$ are PA constraints and $\psi_{i j}$ are formulae of types (F1)-(F3). The automaton $A_{\varphi}$ is defined as $\biguplus_{i \in I} A_{\theta_{i}} \otimes \otimes_{j \in J} A_{\psi_{i j}}$ where $\uplus$ and $\otimes$ are the union and intersection operators on FBCA. The construction of counter automata $A_{\psi_{i j}}$ for the formulae $\psi_{i j}$ of type (F1)-(F3) relies on the definition of the constraint graphs in Section 4.2. Namely, each accepting run of $A_{\psi_{i j}}$ gives a consistent valuation of the constraint graph of $\psi_{i j}$.

Counter Automata Templates. To simplify the definition of counter automata, we note that each constraint graph for the basic formulae of type (F1)-(F3) is composed 
of horizontal, vertical, and diagonal edges, which are defined in roughly the same way for all types of formulae (cf. Section 4.2). We take advantage of this fact and we start by defining three types of counter automata templates, which are subsequently used to define the counter automata for the basic formulae 3 More precisely, the automata for (F1)-(F3) formulae will be defined as $\otimes$-products of particular instances of the automata templates for the horizontal, vertical, and diagonal edges of the appropriate constraint graphs. In the following definitions, we assume the existence of a special counter $x_{\tau}$ (tick) incremented by each transition rule, i.e., we suppose that the constraint $x_{\tau}^{\prime}=x_{\tau}+1$ is implicitly in conjunction with each formula labelling a transition rule. Intuitively, the role of the $x_{\tau}$ counter is to synchronise all automata composed by the $\otimes$-product on a common current position.

The template for the horizontal edges. Let $a$ be an array symbol, dir $\in\{$ left,right, bi $\}$ be a direction parameter, and $\phi$ be a formula on array-bound variables. Let $\mathbf{x}_{\mathbf{k}}$ be the set $\left\{x_{k} \mid k \in F V(\phi)\right\}$. We define the template $H(a, \operatorname{dir}, \phi)=\langle\mathbf{x}, Q, L, R, \rightarrow\rangle$ where:

- $\mathbf{x}=\left\{x_{a}\right\} \cup \mathbf{x}_{\mathbf{k}}$. These counters will have the same names in all instances of $H$.

- $Q=\left\{q_{L}, q_{R}, p_{L}, p_{R}\right\}$. The control states are required to have fresh names in every instance of $H . L=\left\{q_{L}, p_{L}\right\}$ and $R=\left\{q_{R}, p_{R}\right\}$.

- $q_{L} \stackrel{\xi}{\longrightarrow} q_{L}, q_{R} \stackrel{\xi}{\longrightarrow} q_{R}, q_{L} \stackrel{\phi\left(\mathbf{x}_{\mathbf{k}}\right) \wedge \xi}{\longrightarrow} q_{R}, p_{L} \stackrel{\top}{\longrightarrow} p_{L}, p_{R} \stackrel{\top}{\longrightarrow} p_{R}$, and $p_{L} \stackrel{\neg \phi\left(\mathbf{x}_{\mathbf{k}}\right)}{\longrightarrow} p_{R}$.

In the above, $\phi\left(\mathbf{x}_{\mathbf{k}}\right)$ is the formula obtained by replacing each occurrence of an array-bound variable $k \in F V(\phi)$ by its corresponding counter $x_{k}$. The formula $\xi\left(x_{a}, x_{a}^{\prime}\right)$ is $x_{a}-x_{a}^{\prime} \leq 0$ if $d i r=$ right, $x_{a}^{\prime}-x_{a} \leq 0$ if $d i r=$ left, and $x_{a}^{\prime}=x_{a}$ if $d i r=\mathrm{bi}$. Moreover, for each transition rule, we assume the conjunction $\bigwedge_{k \in F V(\phi)} x_{k}^{\prime}=x_{k}$ to be added implicitly to the labelling formula, i.e., the value of an $x_{k}$ counter stays constant throughout a run.

If the formula $\phi$ holds for a given valuation of the parameters $\mathbf{x}_{\mathbf{k}}$, then any accepting run of (any instance of) $H$ visits $q_{L}$ infinitely often on the left and $q_{R}$ infinitely often on the right. Otherwise, if $\phi$ does not hold for the given valuation of $\mathbf{x}_{\mathbf{k}}$, the instance automata have a run that goes infinitely often through $p_{L}$ on the left and through $p_{R}$ on the right. In this case, the automata do not impose any constraints on $x_{a}$.

The template for the diagonal edges. Let $a, b$ be array symbols, $q \in \mathbb{Z}, p, s \in \mathbb{N}^{+}$, $t \in[0, s-1]$, and $\operatorname{dir} \in\{$ left,right $\}$ be a direction parameter. In the following, we refer to the sets $\mathbb{L}=\left\{l_{1}, \ldots, l_{K}\right\}$ and $\mathbb{U}=\left\{u_{1}, \ldots, u_{L}\right\}$ of lower and upper bounds, respectively, where $l_{i}$ and $u_{j}$ are linear combinations of array-bound variables. Let $\mathbf{x}_{\mathbf{k}}=$ $\left\{x_{k} \mid k \in \bigcup_{i=1}^{K} F V\left(l_{i}\right) \cup \bigcup_{j=1}^{L} F V\left(u_{j}\right)\right\}$. Further, we assume that $\mathbb{L} \cup \mathbb{U} \neq \emptyset$ and we deal with the case of $\mathbb{L} \cup \mathbb{U}=\emptyset$ later on. We define the template $D(a, b, p, q, s, t, \mathbb{L}, \mathbb{U}$, dir $)=$ $\langle\mathbf{x}, Q, L, R, \rightarrow\rangle$ where:

- $\mathbf{x}=\left\{x_{a}, x_{b}\right\} \cup \mathbf{x}_{\mathbf{k}} \cup\left\{x_{i} \mid 1 \leq i<p\right\}$. The counters $x_{a}, x_{b}$, and $\mathbf{x}_{\mathbf{k}}$ will have the same names in all instances of $D$. On the other hand, the counters $x_{i}, 1 \leq i<p$, will have fresh names in every instance of $D$. The $x_{i}$ counters are used for splitting

${ }^{3}$ By a template, we mean a class of counter automata which all share the same structure. 
diagonal edges that span over more than one position into series of diagonal edges connecting only adjacent positions 4

- $Q=\left\{q_{L}, q_{R}\right\} \cup\left\{q_{i} \mid 0 \leq i<s\right\} \cup\left\{q_{i}^{j} \mid 0 \leq j<s, j+1 \leq i<j+p\right\}$. The control states are required to have fresh names in every instance of $D$. Let $L=\left\{q_{L}\right\} \cup$ $\left\{q_{i} \mid 0 \leq i<s\right\}$ and $R=\left\{q_{R}\right\} \cup\left\{q_{i} \mid 0 \leq i<s\right\}$.

- $q_{L} \stackrel{\top}{\longrightarrow} q_{L}, q_{R} \stackrel{\top}{\longrightarrow} q_{R}$, and $q_{L} \stackrel{\neg\left(\exists i \cdot \wedge_{l \in \mathbb{L}} i \geq l\left(\mathbf{x}_{\mathbf{k}}\right) \wedge \wedge_{u \in \mathbb{U}} i \leq u\left(\mathbf{x}_{\mathbf{k}}\right) \wedge i \equiv_{s} t\right)}{\longrightarrow} q_{R}$.

- $q_{L} \stackrel{\wedge_{l \in \mathbb{L}} x_{\tau} \geq l\left(\mathbf{x}_{\mathbf{k}}\right)-1 \wedge\left(\bigvee_{l \in \mathbb{L}} x_{\tau}=l\left(\mathbf{x}_{\mathbf{k}}\right)-1\right) \wedge x_{\tau}+1 \equiv_{s} i}{\longrightarrow} q_{i}$ for all $0 \leq i<s$.

- $q_{i} \stackrel{\Lambda_{l \in \mathbb{L}} x_{\tau} \geq l\left(\mathbf{x}_{\mathbf{k}}\right) \wedge \wedge_{u \in \mathbb{U}} x_{\tau}<u\left(\mathbf{x}_{\mathbf{k}}\right) \wedge \xi_{i}\left[x_{a} / x_{0}, x_{b} / x_{p}\right]}{\longrightarrow} q_{(i+1)} \bmod s$ for all $0 \leq i<s$.

- $q_{i} \stackrel{\bigvee_{u \in \mathbb{U}} x_{\tau}=u\left(\mathbf{x}_{\mathbf{k}}\right) \wedge x_{\tau} \equiv_{s} i \wedge \xi_{i}\left[x_{a} / x_{0}, x_{b} / x_{p}\right]}{\longrightarrow} q_{i+1}^{i}$ for all $0 \leq i<s$.

- $q_{i} \stackrel{\bigvee_{u \in \mathbb{U}} x_{\tau}=u\left(\mathbf{x}_{\mathbf{k}}\right) \wedge x_{\tau} \equiv_{s} i \wedge \xi_{i}\left[x_{a} / x_{0}, x_{b} / x_{p}\right]}{\longrightarrow} q_{R}$ for all $0 \leq i<s$ if $p=1$.

- $q_{i}^{j} \stackrel{\xi_{i}\left[x_{a} / x_{0}, x_{b} / x_{p}\right]}{\longrightarrow} q_{i+1}^{j}$ for all $0 \leq j<s, j<i<j+p-1$.

$-q_{j+p-1}^{j} \stackrel{\xi_{i}\left[x_{a} / x_{0}, x_{b} / x_{p}\right]}{\longrightarrow} q_{R}$ for all $0 \leq j<s$ if $p>1$.

In the above, $l\left(\mathbf{x}_{\mathbf{k}}\right)$ and $u\left(\mathbf{x}_{\mathbf{k}}\right)$ denote the expressions $l$ and $u$ in which each occurrence of an array-bound variable $k$ is replaced by its corresponding parameter $x_{k}$. As before, for each transition rule, we assume the conjunction $\bigwedge_{k \in F V(\phi)} x_{k}^{\prime}=x_{k}$ to be added implicitly to the labelling formula, i.e., we require that the value of an $x_{k}$ counter stays constant throughout the run. The formulae $\xi_{i}$ are defined as follows:

- if dir = right, $\xi_{i}=\bigwedge_{k \in K_{i}} x_{k}-x_{k+1}^{\prime} \leq \alpha_{k}$ for $K_{i}=\left\{k \mid 0 \leq k<p, i \equiv_{s} k+t\right\}$, $\alpha_{0}=q$, and $\alpha_{k}=0, k>0$,

- if dir = left, $\xi_{i}=\bigwedge_{k \in K_{i}} x_{k-1}^{\prime}-x_{k} \leq \alpha_{k}, K_{i}=\left\{k \mid 1 \leq k \leq p, k+i \equiv_{s} t\right\}, \alpha_{1}=q$, and $\alpha_{k}=0, k>1$.

Finally, for the case $\mathbb{L}=\mathbb{U}=\emptyset$, we define any instance of $D(a, b, p, q, s, t, \emptyset, \emptyset$, dir $)$ to be $A_{1} \otimes A_{2}$ where $A_{1}$ is an instance of $D(a, b, p, q, s, t, \emptyset,\{0\}, d i r)$ and $A_{2}$ is an instance of $D(a, b, p, q, s, t,\{0\}, \emptyset$, dir $)$.

The construction can be understood by considering an accepting run of (any instance of) $D$. Let us consider the case in which there exists a value $i$ in between the bounds that satisfies also the modulo constraint. If this is not the case, there will be an accepting run that takes the transition $q_{L} \stackrel{\neg\left(\exists i \cdot \bigwedge_{l \in \mathbb{L}} i \geq l\left(\mathbf{x}_{\mathbf{k}}\right) \wedge \wedge_{u \in \mathbb{U}} i \leq u\left(\mathbf{x}_{\mathbf{k}}\right) \wedge i \equiv_{s} t\right)}{\longrightarrow} q_{R}$ exactly once.

Since the run is accepting, it must visit a state from $L$ infinitely often on the left, and a state from $R$ infinitely often on the right. There are three cases: (1) $\mathbb{L} \neq \emptyset$ and $\mathbb{U} \neq \emptyset$, (2) $\mathbb{L}=\emptyset$ and $\mathbb{U} \neq \emptyset$, and (3) $\mathbb{L} \neq \emptyset$ and $\mathbb{U}=\emptyset$. In the case (1), a bi-infinite run will visit $q_{L}$ infinitely often on the left and $q_{R}$ infinitely often on the right. Notice that the run

\footnotetext{
${ }^{4}$ For instance, the constraint $a[i]-b[i+3] \leq 5$ can be split to $a[i]-x_{1}[i+1] \leq 5, x_{1}[i+1]-x_{2}[i+$ $2] \leq 0$, and $x_{2}[i+2]-b[i+3] \leq 0$. The constraints for array values of neighbouring indices can then be conveniently expressed by using the current and future values of the appropriate counters (e.g., for our example constraint, $x_{a}-x_{1}^{\prime} \leq 5, x_{1}-x_{2}^{\prime} \leq 0$, and $x_{2}-x_{b}^{\prime} \leq 0$, which of course appear on subsequent transitions of the appropriate FBCA).
} 


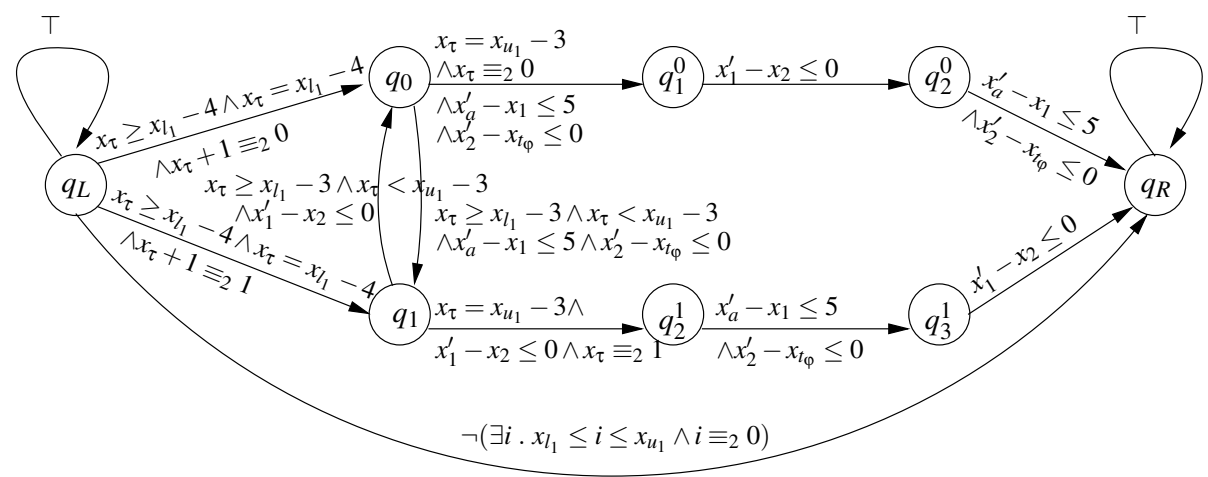

Fig. 3. The FBCA for the diagonal edges in the formula $\varphi: \forall i, j . l_{1} \leq i \leq u_{1} \wedge l_{2} \leq j \leq u_{2} \wedge i-j \leq$ $3 \wedge i \equiv_{2} 0 \wedge j \equiv_{2} 1 \rightarrow a[i]-b[j] \leq 5$ from Fig. 2(a) obtained as $D\left(a, t_{\varphi}, 3,5,2,0-3,\left\{l_{1}-3\right\},\left\{u_{1}-\right.\right.$ $3\}$, left). To understand the formula $\xi_{0}$ on the transition from $q_{0}$ to $q_{1}$, note that the constraint $i \equiv_{s} k+t$ in the definition of the set $K_{0}$ instantiates to $0 \equiv_{2} k-3$, and hence $K_{0}=\{1,3\}$. A similar reasoning applies for the other transitions.

cannot visit the loop $q_{0} \rightarrow \ldots \rightarrow q_{s-1}$ infinitely often due to the presence of both lower and upper bounds on $x_{\tau}$. In the case (2), the run cannot take any of the transitions $q_{L} \rightarrow$ $q_{i}, 0 \leq i<s$, due to the emptiness of $\mathbb{L}$, which makes the guard unsatisfiable. Hence, the only possibility for an accepting bi-infinite run is to visit the states $q_{0} \rightarrow \ldots \rightarrow q_{s-1}$ infinitely often on the left. Due to the presence of the upper bound on $x_{\tau}$, the run cannot stay forever inside this loop and must exit via one of the $q_{i} \rightarrow q_{i+1}^{i}$ (or $q_{i} \rightarrow q_{R}$ for $p=1$ ) transitions, getting trapped into $q_{R}$ on the right. Case (3) is symmetric to (2).

Note that, in all cases, due to the modulo tests on $x_{\tau}$ in the entry and exit of the main loop $q_{0} \rightarrow \ldots \rightarrow q_{s-1}$ on any accepting run, whenever a state $q_{i}, 0 \leq i<s$, is visited, the value of the $x_{\tau}$ counter must equal $i$ modulo $s$. Note also that the role of the $q_{i}^{j}$ states is to describe constraints corresponding to edges that start inside the given interval bounds and lead above its upper bound (or vice versa). The number of such edges is bounded. We do not use the same construction at the beginning of the interval as the templates are applied such that none of the edges represented goes below the lower bounds.

Template for the vertical edges. Let $a, b$ be array symbols, $q$ a linear combination of array-bound variables, $p, s \in \mathbb{N}^{+}$, and $t \in[0, s-1]$. We again refer to the sets $\mathbb{L}=$ $\left\{l_{1}, \ldots, l_{K}\right\}$ and $\mathbb{U}=\left\{u_{1}, \ldots, u_{L}\right\}$ of lower and upper bounds, respectively, where $l_{i}$ and $u_{j}$ are linear combinations of array-bound variables. Also, let $\mathbf{x}_{\mathbf{k}}=\left\{x_{k} \mid k \in \bigcup_{i=1}^{K} F V\left(l_{i}\right)\right.$ $\left.\cup \bigcup_{j=1}^{L} F V\left(u_{j}\right)\right\}$. Further, we assume that $\mathbb{L} \cup \mathbb{U} \neq \emptyset$ and we deal with the case of $\mathbb{L} \cup \mathbb{U}=\emptyset$ later on. We define the template $V(a, b, p, q, s, t, \mathbb{L}, \mathbb{U})=\langle\mathbf{x}, Q, L, R, \rightarrow\rangle$ where:

- $\mathbf{x}=\left\{x_{a}, x_{b}\right\} \cup \mathbf{x}_{\mathbf{k}}$. The counters $x_{a}, x_{b}, \mathbf{x}_{\mathbf{k}}$ have the same names in all instances of $V$.

- $Q=\left\{q_{L}, q_{R}\right\} \cup\left\{q_{i} \mid 0 \leq i<s\right\}$. The control states are required to have fresh names in every instance of $V . L=\left\{q_{L}\right\} \cup\left\{q_{i} \mid 0 \leq i<s\right\}$ and $R=\left\{q_{R}\right\} \cup\left\{q_{i} \mid 0 \leq i \leq s\right\}$.

- $q_{L} \stackrel{\top}{\longrightarrow} q_{L}, q_{R} \stackrel{\top}{\longrightarrow} q_{R}$, and $q_{L} \stackrel{\neg\left(\exists i \cdot \wedge_{l \in \mathbb{L}} i \geq l\left(\mathbf{x}_{\mathbf{k}}\right) \wedge \wedge_{u \in \mathbb{U}} i \leq u\left(\mathbf{x}_{\mathbf{k}}\right) \wedge i \equiv_{s} t\right)}{\longrightarrow} q_{R}$. 


$$
\begin{aligned}
& -q_{L} \stackrel{\Lambda_{l \in \mathbb{L}} x_{\tau} \geq l\left(\mathbf{x}_{\mathbf{k}}\right)-1 \wedge \bigvee_{l \in \mathbb{L}} x_{\tau}+1=l\left(\mathbf{x}_{\mathbf{k}}\right) \wedge x_{\tau}+1 \equiv_{s} i}{\longrightarrow} q_{i}, 0 \leq i<s . \\
& -q_{i} \stackrel{\bigwedge_{l \in \mathbb{L}} x_{\tau} \geq l\left(\mathbf{x}_{\mathbf{k}}\right) \wedge \wedge_{u \in \mathbb{U}} x_{\tau}<u\left(\mathbf{x}_{\mathbf{k}}\right) \wedge x_{a}-x_{b} \leq q\left(\mathbf{x}_{\mathbf{k}}\right)}{\longrightarrow} q_{(i+1)} \bmod s, 0 \leq i<s \text { and } i \equiv_{s} t . \\
& -q_{i} \stackrel{\bigwedge_{l \in \mathbb{L}} x_{\tau} \geq l\left(\mathbf{x}_{\mathbf{k}}\right) \wedge \wedge_{u \in \mathbb{U}} x_{\tau}<u\left(\mathbf{x}_{\mathbf{k}}\right)}{\longrightarrow} q_{(i+1)} \bmod s, 0 \leq i<s \text { and } i \not \equiv_{s} t . \\
& -q_{i} \stackrel{\bigvee_{u \in \mathbb{U}} x_{\tau}=u\left(\mathbf{x}_{\mathbf{k}}\right) \wedge x_{\tau} \equiv_{s} i \wedge x_{a}-x_{b} \leq q\left(\mathbf{x}_{\mathbf{k}}\right)}{\longrightarrow} q_{R}, 0 \leq i<s \text { and } i \equiv_{s} t . \\
& -q_{i} \stackrel{\bigvee_{u \in \mathbb{U}} x_{\tau}=u\left(\mathbf{x}_{\mathbf{k}}\right) \wedge x_{\tau} \equiv_{s} i}{\longrightarrow} q_{R}, 0 \leq i<s \text { and } i \not{ }_{s} t .
\end{aligned}
$$

In the above, $l\left(\mathbf{x}_{\mathbf{k}}\right), u\left(\mathbf{x}_{\mathbf{k}}\right)$, and $q\left(\mathbf{x}_{\mathbf{k}}\right)$ denote the expressions $l, u$, and $q$ where each occurrence of an array-bound variable $k$ is replaced by the parameter $x_{k}$. As before, we assume that for each transition rule the conjunction $\bigwedge_{k \in F V(\phi)} x_{k}^{\prime}=x_{k}$ is added implicitly to the labelling formula, i.e., the value of an $x_{k}$ counter stays constant throughout the run. Finally, if $\mathbb{L}=\mathbb{U}=\emptyset$, we define any instance of $V(a, b, p, q, s, t, \emptyset, \emptyset)$ as $A_{1} \otimes A_{2}$ where $A_{1}$ is an instance of $V(a, b, p, q, s, t, \emptyset,\{0\})$ and $A_{2}$ is an instance of $V(a, b, p, q, s, t,\{0\}, \emptyset)$. The intuition behind the construction of $V$ is similar to the one of $D$.

\subsection{Counter Automata for Basic Formulae}

We are now ready to define the construction of FBCA for the basic formulae. This is done by composing instances of templates using the $\otimes$ operator for intersection (cf. Section 2). For space reasons, we only give here the construction of the FBCA for (F3) formulae. The formulae of type $(\mathrm{F} 1),(\mathrm{F} 2)$, and PA constraints on array-bound variables are treated analogously in [8]. Let $\varphi$ be an (F3)-type formula

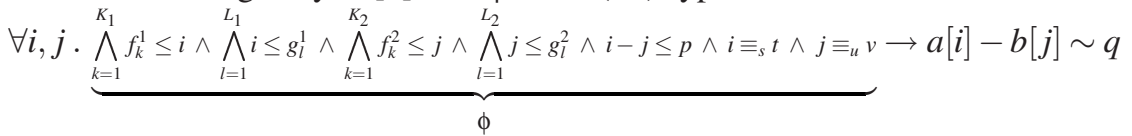

where $0 \leq s<t$ and $0 \leq u<v$. Let $\mathbb{L}_{i}=\left\{f_{1}^{i}, \ldots, f_{K_{i}}^{i}\right\}$ and $\mathbb{U}_{i}=\left\{g_{1}^{i}, \ldots, g_{L_{i}}^{i}\right\}$ for $i=1,2$, respectively. By $\phi$, we denote the precondition of $\varphi$. The automaton $A_{\varphi}$ is defined as $A_{\varphi}=A_{1} \otimes A_{2} \otimes A_{3}$ where $A_{1}, A_{2}, A_{3}$ are instantiated according to Table 1

\subsection{Assembling Automata for Entire Normalised Formulae}

Given a formula $\varphi(\mathbf{k}, \mathbf{a})$ which is a positive boolean combination of formulae of types (F1)-(F3) and PA constraints on the array-bound variables $\mathbf{k}$, let $A_{\varphi}$ be the automaton defined inductively on the structure of $\varphi$ as follows:

- if $\varphi$ is of type (F1)-(F3), or a PA constraint on $\mathbf{k}$, then $A_{\varphi}$ is as in Section 4.4,

- if $\varphi=\psi_{1} \wedge \psi_{2}$, then $A_{\varphi}=A_{\psi_{1}} \otimes A_{\psi_{2}}$,

- if $\varphi=\psi_{1} \vee \psi_{2}$, then $A_{\varphi}=A_{\psi_{1}} \uplus A_{\psi_{2}}$.

Let $r \in \mathcal{R}\left(A_{\varphi}\right)$ be an accepting run of $A_{\varphi}$ and $\delta(r)=\operatorname{val}(r(0))\left(x_{\tau}\right)$ be the value of the $x_{\tau}$ (tick) counter at position 0 on $r$. We denote by $\eta(r)=r \circ \sigma^{-\delta(r)}$ the centered run obtained from $r$ by shifting it such that the value of $x_{\tau}$ at position 0 is also 0 . By Lemma $1 r$ is an accepting run of $A_{\varphi}$ if and only if $\eta(r)$ is. Notice that $r$ induces the 
Table 1. The instantiation table for (F3) formulae. Note that in some lines, we shift the original bounds appearing in the formula in order to be able to re-use the prepared templates that do not explicitly deal with edges leaving from within the given bounds and going below the lower bound. Due to the way the templates are constructed, the shifting preserves the semantics of the formula - instead of edges going below the lower bound of a certain interval, we obtain the same edges just going above the upper bound of the shifted interval, which our templates are prepared for. Given a set of integers $S$ and an integer $p$, we use the notation $S+p$ for $\{s+p \mid s \in S\}$.

\begin{tabular}{|l|l|l|l|l|}
\hline $\mathrm{p}$ & $\sim$ & \multicolumn{1}{|c|}{$A_{1}$} & \multicolumn{1}{c|}{$A_{2}$} & \multicolumn{1}{c|}{$A_{3}$} \\
\hline \hline$\infty$ & $\leq$ & $V\left(a, t_{\varphi}, q, s, t, \mathbb{L}_{1}, \mathbb{U}_{1}\right)$ & $H\left(t_{\varphi}\right.$, bi $\left., \exists i, j . \phi\right)$ & $V\left(t_{\varphi}, b, 0, u, v, \mathbb{L}_{2}, \mathbb{U}_{2}\right)$ \\
\hline$\infty$ & $\geq$ & $V\left(b, t_{\varphi},-q, u, v, \mathbb{L}_{2}, \mathbb{U}_{2}\right)$ & $H\left(t_{\varphi}\right.$, bi, $\left.\exists i, j . \phi\right)$ & $V\left(t_{\varphi}, a, 0, s, t, \mathbb{L}_{1}, \mathbb{U}_{1}\right)$ \\
\hline 0 & $\leq$ & $V\left(a, t_{\varphi}, q, s, t, \mathbb{L}_{1}, \mathbb{U}_{1}\right)$ & $H\left(t_{\varphi}\right.$, right, $\left.\exists i, j . \phi\right)$ & $V\left(t_{\varphi}, b, 0, u, v, \mathbb{L}_{2}, \mathbb{U}_{2}\right)$ \\
\hline 0 & $\geq$ & $V\left(b, t_{\varphi},-q, u, v, \mathbb{L}_{2}, \mathbb{U}_{2}\right)$ & $H\left(t_{\varphi}\right.$, left $\left., \exists i, j . \phi\right)$ & $V\left(t_{\varphi}, a, 0, s, t, \mathbb{L}_{1}, \mathbb{U}_{1}\right)$ \\
\hline$>0$ & $\leq$ & $D\left(a, t_{\varphi}, p, q, s, t-p, \mathbb{L}_{1}-p, \mathbb{U}_{1}-p\right.$, left $)$ & $H\left(t_{\varphi}\right.$, right $\left., \exists i, j . \phi\right)$ & $V\left(t_{\varphi}, b, 0, u, v, \mathbb{L}_{2}, \mathbb{U}_{2}\right)$ \\
\hline$>0$ & $\geq$ & $D\left(b, t_{\varphi}, p,-q, u, v, \mathbb{L}_{2}, \mathbb{U}_{2}\right.$, right $)$ & $H\left(t_{\varphi}\right.$, left $\left., \exists i, j . \phi\right)$ & $V\left(t_{\varphi}, a, 0, s, t, \mathbb{L}_{1}, \mathbb{U}_{1}\right)$ \\
\hline$<0$ & $\leq$ & $D\left(a, t_{\varphi},-p, q, s, t, \mathbb{L}_{1}, \mathbb{U}_{1}\right.$, right $)$ & $H\left(t_{\varphi}\right.$, right, $\left.\exists i, j . \phi\right)$ & $V\left(t_{\varphi}, b, 0, u, v, \mathbb{L}_{2}, \mathbb{U}_{2}\right)$ \\
\hline$<0$ & $\geq$ & $D\left(b, t_{\varphi},-p,-q, u, v+p, \mathbb{L}_{2}+p, \mathbb{U}_{2}+p\right.$, left $)$ & $H\left(t_{\varphi}\right.$, left $\left., \exists i, j . \phi\right)$ & $V\left(t_{\varphi}, a, 0, s, t, \mathbb{L}_{1}, \mathbb{U}_{1}\right)$ \\
\hline
\end{tabular}

following valuations on $\mathbf{k}$ and $\mathbf{a}$, respectively: $\mathbf{1}_{r}(k)=\operatorname{val}(\eta(r)(0))\left(x_{k}\right)$ for all $k \in \mathbf{k}$, and $\mu_{r}(a)(i)=\operatorname{val}(\eta(r)(i))\left(x_{a}\right)$ for all $a \in \mathbf{a}$ and $i \in \mathbb{Z}$.

For an arbitrary valuation $v \in \mathcal{V}\left(A_{\varphi}\right)$, there exists $r \in \mathcal{R}\left(A_{\varphi}\right)$ such that $v=\operatorname{val}(r)$. Let $M_{\varphi}(v)=\left\langle\mathbf{l}_{r}, \mu_{r}\right\rangle$ be the valuation of the free variables in $\varphi$ that corresponds to $r$. One can see now that $M_{\varphi}$ defines a function $M_{\varphi}: \mathcal{V}\left(A_{\varphi}\right) \rightarrow(\mathbf{k} \mapsto \mathbb{Z}) \times\left(\mathbf{a} \mapsto{ }^{\omega} \mathbb{Z}^{\omega}\right)[5$

Theorem 1. Let $\varphi(\mathbf{k}, \mathbf{a})$ be a positive boolean combination of formulae of types $(F 1)$ (F3) and PA constraints on the array-bound variables $\mathbf{k}$, and $A_{\varphi}$ be the automaton defined in the previous. Then, $M_{\varphi}\left(\mathcal{V}\left(A_{\varphi}\right)\right)=[[\varphi]]$.

The proof is by induction on the structure of $\varphi$. For the base case, we use the correspondence between models and constraint graphs of formulae (F1)-(F3) (Lemma 6) The inductive step follows as a consequence of the fact that the class of FBCA is closed under union and intersection (Lemma 3). The main result of the paper is the following:

\section{Corollary 1. The logic LIA is decidable.}

The proof of Corollary 1 uses the normalisation step (cf. Lemma 5) to rewrite any formula of LIA into the form (NF) and applies Theorem 1 to the matrix of the formula (i.e., the formula obtained by skipping the existential quantifier prefix).

\section{Conclusions and Future Work}

We have presented a new decidable logic for reasoning about properties of programs with integer arrays. This logic allows one to relate adjacent array values as well as to

\footnotetext{
${ }^{5}$ By definition, for each $v \in \mathcal{V}\left(A_{\varphi}\right)$, there exist valuations $\mathrm{t}_{r}$ and $\mu_{r}$, so $M_{\varphi}$ is defined for all $v \in \mathcal{V}\left(A_{\varphi}\right)$. Let $r_{1}, r_{2} \in \mathcal{R}\left(A_{\varphi}\right)$ be two runs such that $\operatorname{val}\left(r_{1}\right)=\operatorname{val}\left(r_{2}\right)=v$. We have $\delta\left(r_{1}\right)=$ $\delta\left(r_{2}\right)$, therefore $\eta\left(r_{1}\right)=\eta\left(r_{2}\right)$, which leads to $\mathrm{l}_{r_{1}}=\mathfrak{1}_{r_{2}}$ and $\mu_{r_{1}}=\mu_{r_{2}}$.
} 
express periodic facts relating all values situated at equidistant positions. We have established decidability of this logic by following the automata-theoretic approach. To this end, we have defined a new class of Büchi automata with counters, for which emptiness is decidable. We translate each formula into a corresponding automaton of this kind and transform deciding satisfiability of the formula to deciding emptiness of the automaton.

Future work will include the study of the complexity of our decision procedure and its implementation. We furthermore plan to develop invariant generation methods in order to give automatic correctness proofs for programs with integer arrays.

\section{References}

1. Armando, A., Ranise, S., Rusinowitch, M.: Uniform Derivation of Decision Procedures by Superposition. In: Fribourg, L. (ed.) CSL 2001. LNCS, vol. 2142, p. 2001. Springer, Heidelberg (2001)

2. Arons, T., Pnueli, A., Ruah, S., Xu, J., Zuck, L.: Parameterized Verification with Automatically Computed Inductive Assertions. In: Berry, G., Comon, H., Finkel, A. (eds.) CAV 2001. LNCS, vol. 2102, Springer, Heidelberg (2001)

3. Bouajjani, A., Jurski, Y., Sighireanu, M.: A Generic Framework for Reasoning About Dynamic Networks of Infinite-State Processes. In: Grumberg, O., Huth, M. (eds.) TACAS 2007. LNCS, vol. 4424, Springer, Heidelberg (2007)

4. Bozga, M., Iosif, R., Lakhnech, Y.: Flat Parametric Counter Automata. In: Bugliesi, M., Preneel, B., Sassone, V., Wegener, I. (eds.) ICALP 2006. LNCS, vol. 4052, Springer, Heidelberg (2006)

5. Bradley, A.R., Manna, Z., Sipma, H.B.: What's Decidable About Arrays? In: Emerson, E.A., Namjoshi, K.S. (eds.) VMCAI 2006. LNCS, vol. 3855, Springer, Heidelberg (2005)

6. Comon, H., Jurski, Y.: Multiple Counters Automata, Safety Analysis and Presburger Arithmetic. In: Vardi, M.Y. (ed.) CAV 1998. LNCS, vol. 1427, Springer, Heidelberg (1998)

7. Ghilardi, S., Nicolini, E., Ranise, S., Zucchelli, D.: Decision Procedures for Extensions of the Theory of Arrays. Annals of Mathematics and Artificial Intelligence 50 (2007)

8. Habermehl, P., Iosif, R., Vojnar, T.: What else is decidable about integer arrays? Technical Report TR-2007-8, Verimag (2007)

9. Jaffar, J.: Presburger Arithmetic with Array Segments. Inform. Proc. Letters 12 (1981)

10. King, J.: A Program Verifier. PhD thesis, Carnegie Mellon University (1969)

11. Mateti, P.: A Decision Procedure for the Correctness of a Class of Programs. Journal of the ACM 28(2) (1980)

12. McCarthy, J.: Towards a Mathematical Science of Computation. In: IFIP Congress (1962)

13. Minsky, M.L.: Computation: Finite and Infinite Machines. Prentice-Hall, Inc., Englewood Cliffs (1967)

14. Nivat, M., Perrin, D.: Ensembles reconnaissables de mots biinfinis. Canad. J. Math. 38, 513 537 (1986)

15. Presburger, M.: Über die Vollständigkeit eines gewissen Systems der Arithmetik ganzer Zahlen, in welchem die Addition als einzige Operation hervortritt. In: Comptes Rendus du Premier Congrès des Mathématiciens des Pays Slaves, Warsaw, Poland, pp. 92-101 (1929)

16. Stump, A., Barrett, C.W., Dill, D.L., Levitt, J.R.: A Decision Procedure for an Extensional Theory of Arrays. In: Proc. of LICS 2001 (2001)

17. Suzuki, N., Jefferson, D.: Verification Decidability of Presburger Array Programs. Journal of the ACM 27(1) (1980)

18. Thomas, W.: Automata on Infinite Objects. In: Handbook of Theoretical Computer Science. Formal Models and Semantics, vol. B, Elsevier, Amsterdam (1990) 